\title{
Milliseconds make the difference in the far-from-equilibrium self- assembly of supramolecular chiral nanostructures
}

\author{
Alessandro Sorrenti $^{\mathrm{a}{ }^{\dagger}}$, Romen Rodriguez-Trujillo ${ }^{\mathrm{a}}$, David B. Amabilino ${ }^{\mathrm{a}, \mathrm{b} *}$, Josep Puigmartí- \\ Luis $^{\mathrm{a}, \mathrm{c} *}$ \\ ${ }^{a}$ Institut de Ciència de Materials de Barcelona (ICMAB-CSIC), Campus Universitari de Bellaterra, o8193 \\ Cerdanyola del Vallès, Catalonia, Spain. \\ ${ }^{\mathrm{b}}$ School of Chemistry, The University of Nottingham, University Park, NG7 2RD, UK. \\ ${ }^{c}$ Empa, Swiss Federal Laboratories for Materials Science and Technology, Lerchenfeldstrasse 5, 9014 St. Gallen, \\ Switzerland.
}




\section{Section 1. Experimental Section}

Sample preparation. We studied chiral induction in the formation of surfactant/porphyrin aggregates both by using a microfluidic approach as well as in "bulk" samples conveniently prepared to compare controlled microfluidic diffusion mixing with chaotic mixing.

Microfluidic samples. The microfluidic experiments were carried out using planar devices imprinted in poly(dimethylsiloxane) (PDMS). The microfluidic chips were designed with four inlet channels (a)-(d) and one outlet (see Figure $1 \mathrm{~b}$ in the main text), and were prepared following standard soft lithography techniques. ${ }^{1}$ The length and width of the main channel were $10 \mathrm{~mm}$ and 250 microns, respectively. The use of PDMS is preferable because optical microscopy can be used to assess the progress of the microfluidic mixing in real time and because PDMS-based chips are easily molded.

"Bulk" samples. Laboratory flask chaotic mixing experiments were conducted using standard protocols. For example, for experiments without chiral dopant, samples were prepared by adding $1 \mathrm{ml}$ of $1(40 \mu \mathrm{M})$ and $1 \mathrm{ml}$ of $2(80 \mu \mathrm{M})$ to a glass vial followed by the rapid addition of $1 \mathrm{ml}$ of $\mathrm{HCl}_{(\mathrm{aq})}(2 \mathrm{mM})$ and $1 \mathrm{ml}$ water under gentle shacking. On the other hand, for samples prepared under chaotic mixing with chiral dopant $\mathbf{3}, 1 \mathrm{ml}$ of a solution of $\mathbf{2} / \mathbf{3}$ in a ratio nine to one $(80 \mu \mathrm{M})$ was added to $1 \mathrm{ml}$ of $\mathbf{1}(40 \mu \mathrm{M})$ in a glass vial followed by the addition of $1 \mathrm{ml}$ of $\mathrm{HCl}_{(\mathrm{aq})}(2 \mathrm{mM})$ and $1 \mathrm{ml}$ water and all mixed under gentle shacking. Flask samples for the experiments reported in Figure 2 of the main text were prepared by adding $1 \mathrm{ml}$ of an aqueous solution of 1 to $1 \mathrm{ml}$ of $\mathbf{2} / \mathbf{3}$ solution in $2 \mathrm{mM} \mathrm{HCl}(\mathrm{aq})$, in a glass vial, followed by the addition of $2 \mathrm{ml}$ of water and gentle shacking, for a better comparison with microfluidic sample M5. 


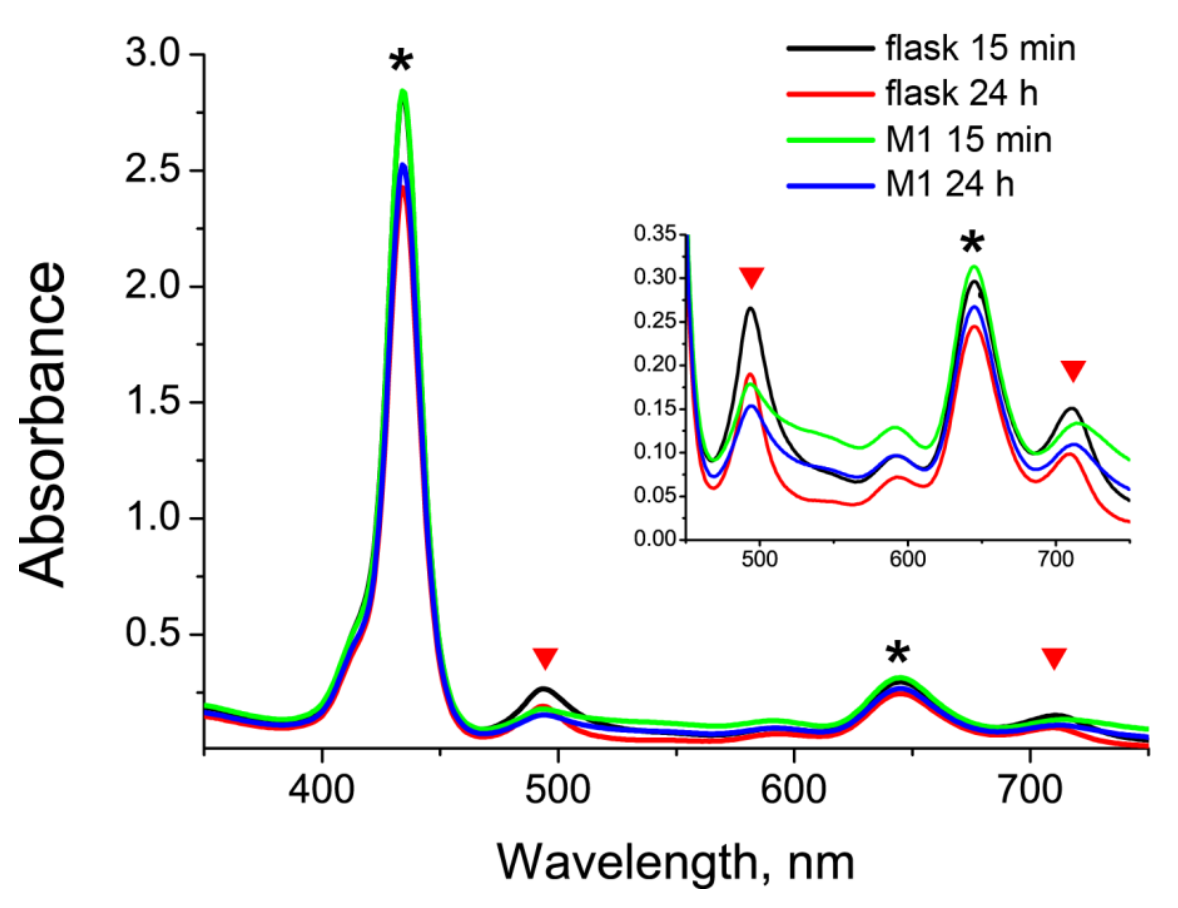

Figure S1. Typical UV-Vis absorption spectra and their time evolution for samples of $\mathbf{2} / \mathbf{1}$ (two to one), [1] = $10 \mu \mathrm{M}$, optical path $=1 \mathrm{~cm}$, prepared by flask mixing (black and red lines) and M1 (green and blue). The black asterisks indicate the Soret $(434 \mathrm{~nm})$ and the main Q-band $(650 \mathrm{~nm})$ of the free diprotonated zwitterionic porphyrin 1. The two red triangles indicate bands at $495 \mathrm{~nm}$ and $716 \mathrm{~nm}$ that are ascribed to Jtype surfactant/porphyrin (2/1) heteroaggregates. ${ }^{2}$ Inset: zoom of the lower energy region $(450 \mathrm{~nm}-750 \mathrm{~nm})$ of the absorption spectrum. 
A

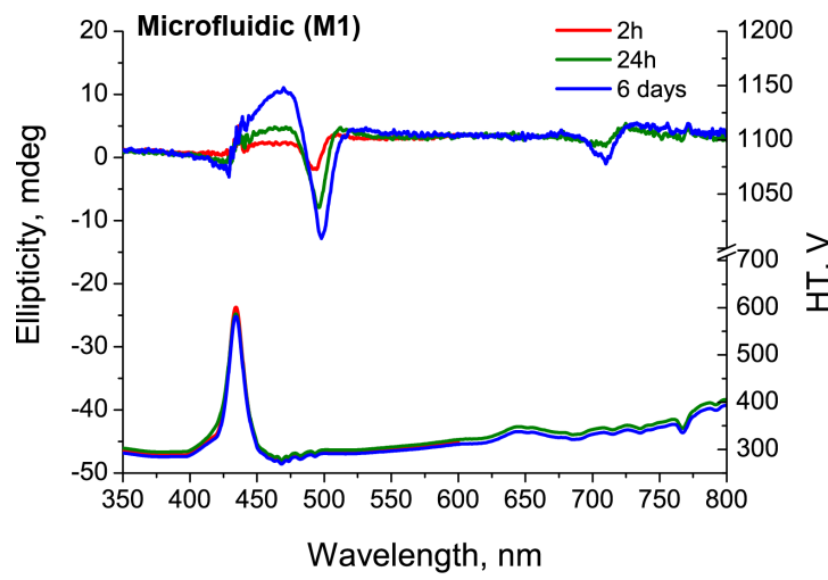

C

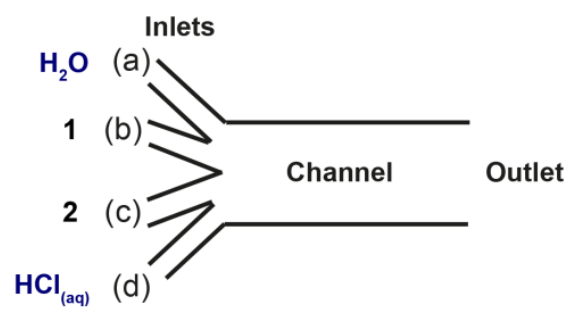

B

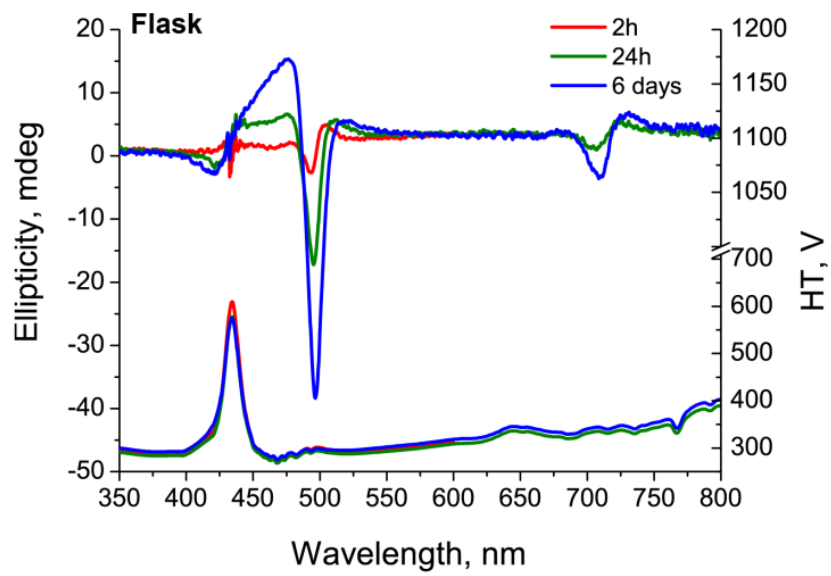

Figure S2. (A) and (B), CD spectra (top) and HT voltage profiles (bottom) at $t=2 h, 24 \mathrm{~h}, 6$ days for samples of $\mathbf{2} / \mathbf{1}$ (at ratio two to one), [1] $=10 \mu \mathrm{M}$, optical path $=1 \mathrm{~cm}$, prepared by microfluidic (M1 samples), (A), and flask mixing, (B). An excitonic CD band (negative couplet) in correspondence with the main absorption band of the surfactant/porphyrin J-aggregates $(\sim 490 \mathrm{~nm})$ is observed for both samples, whose intensity increases with time. In (C), a schematic illustration of the experimental setup used for the synthesis of M1 samples is presented. Namely, aqueous solutions of $1(40 \mu \mathrm{M})$ and $2(80 \mu \mathrm{M})$ were injected in the middle inlet channels (b) and (c), while water and $\mathrm{HCl}_{(\mathrm{aq})}(2 \mathrm{mM})$ were injected through the side inlets (a) and (d), respectively. The flask mixed samples corresponding to the spectra shown in (B) were prepared as explained in Section 1. 
Section 3. Simulations of proton and porphyrin diffusion in the microfluidic chip by COMSOL Multiphysics ${ }^{\text {MM }}$

2D finite element simulations of the diffusion of the proton and the porphyrin inside the microfluidic channel have been performed using a finite element method (FEM) package (i.e. COMSOL Multiphysics ${ }^{\mathrm{TM}}$ 4.3.2.152) incorporating fluid dynamic and chemical transport models. Both, the dimensions of the chip and flow rates in our simulation studies have been fixed to the experimental conditions used in our investigations. That is, the dimensions of the microfluidic channel considered in our finite element simulations are $250 \mu \mathrm{m}$ in width and $1 \mathrm{~cm}$ in length and the flow rates in all four inlet channels are $100 \mu 1 / \mathrm{min}$. Diffusion coefficients for the proton and the porphyrin have been set to $5 \times 10^{-9} \mathrm{~m}^{2} / \mathrm{s}$ and $3 \times 10^{-10} \mathrm{~m}^{2} / \mathrm{s}$, respectively, according to previously reported values. ${ }^{3,4}$ We have considered two different experimental conditions: i) the case where $\mathrm{HCl}_{(\mathrm{aq})}(2 \mathrm{mM})$ is injected in the inlet channel (d), while 1 is injected in the inlet channel (b) (i.e. the two reagent-laden flows are separated by a water stream (Figure S3)), and ii) the case where $\operatorname{HCl}_{(\mathrm{aq})}(2 \mathrm{mM})$ is injected in the microfluidic channel using the inlet channel (c), while $\mathbf{1}$ is injected in the middle inlet channel (b) (i.e., the porphyrin and $\mathrm{HCl}$ streams are adjacent to each other (Figure S4)). In both simulation studies, three positions of the microfluidic channel have been investigated, position 1 (P1), position 2 (P2) and position 3 (P3). P1 is located at $200 \mu \mathrm{m}$ along the $\mathrm{x}$-coordinate of the microfluidic channel, $\mathrm{P} 2$ at $5000 \mu \mathrm{m}$ and $\mathrm{P} 3$ at $9700 \mu \mathrm{m}$. 
A

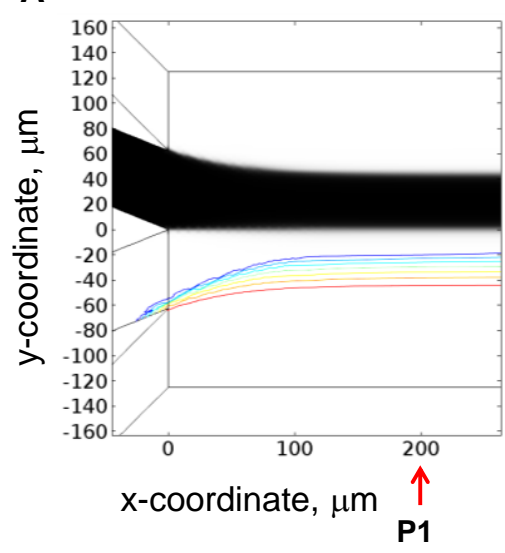

C

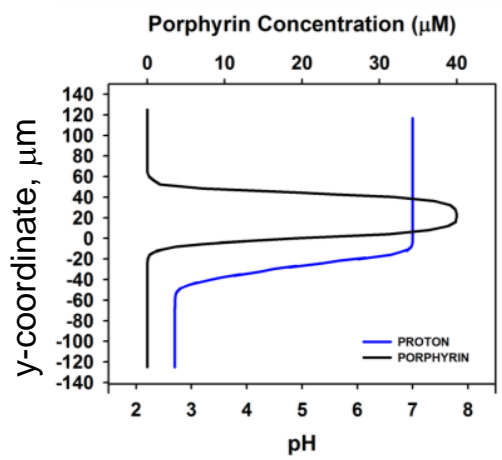

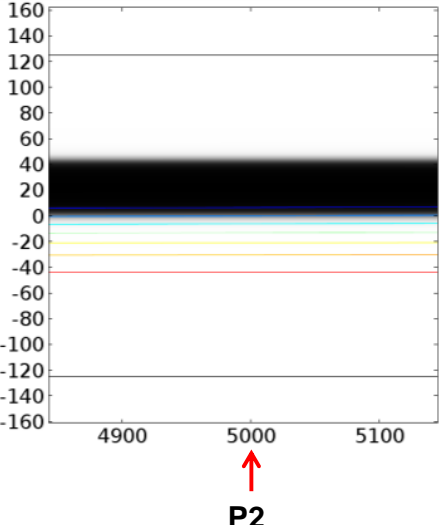

P2
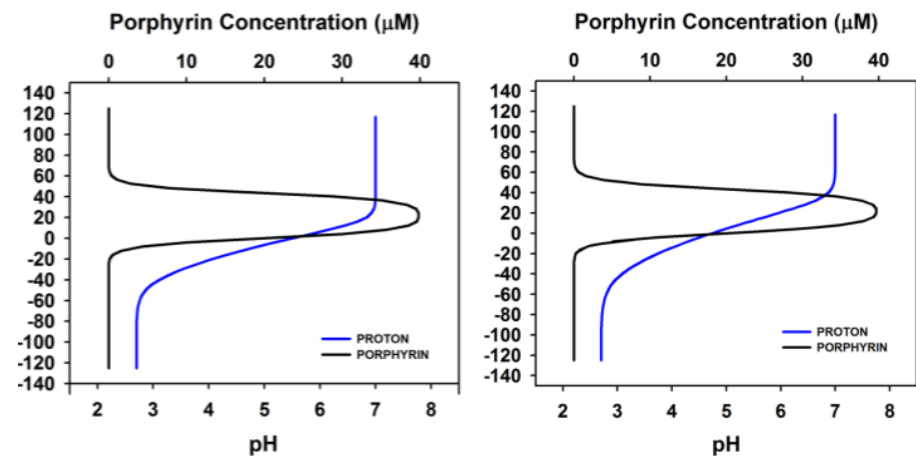

B
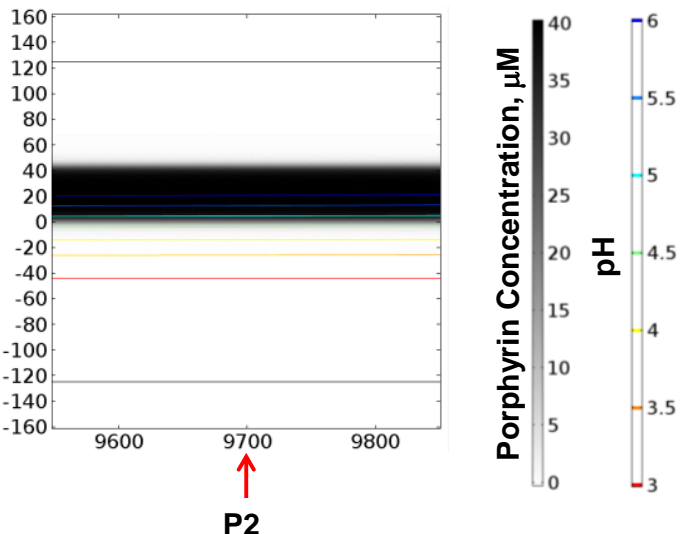

Figure S3. (A) 2D finite element simulations showing the diffusion of $\mathbf{1}$ (black color) and proton (colored lines) at P1 (left image), P2 (middle image) and P3 (right image). In (B), color legends for porphyrin concentration and $\mathrm{pH}$ are shown. In $(\mathrm{C})$, one-dimensional concentration profiles of the porphyrin (black line) and $\mathrm{pH}$ (blue line) at P1, P2 and P3. The sections in (B) are made transversal to the direction of the flow, i.e. along the Y-coordinate axis. The simulation results clearly show that the presence of the water stream coflowing between the 1-laden flow and $\mathrm{HCl}$ stream avoids protonation of the porphyrin inside the microfluidic channel. As shown in Figure S3 (C), the pH at P1, P2 and P3 is well above four when compared to the porphyrin concentration profile, and hence, porphyrin protonation can only occur outside the microfluidic channel under non-laminar flow conditions. The latter, can be safely stated, taking into account that the pKa of pure $\mathrm{H}_{2} \mathrm{TPPS}_{4}$ is 4.8 . ${ }^{5,6}$ 
A
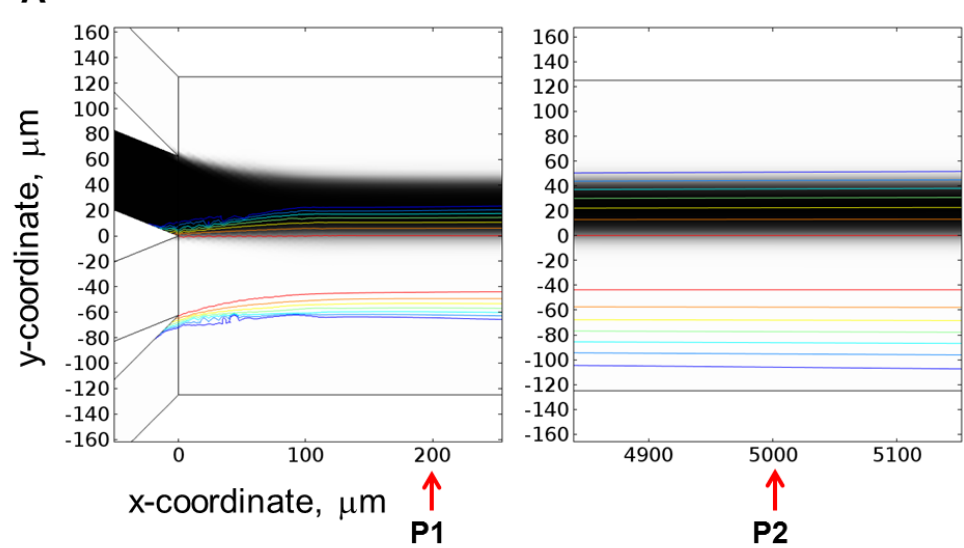

C

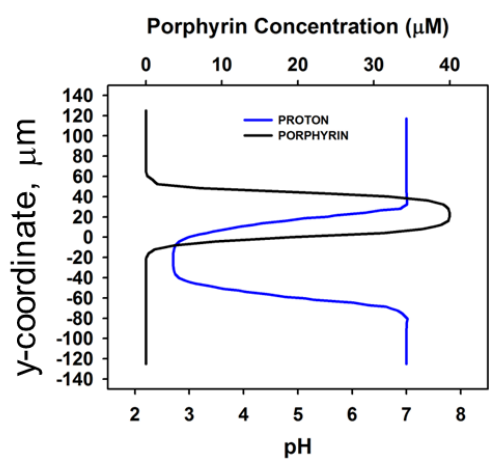

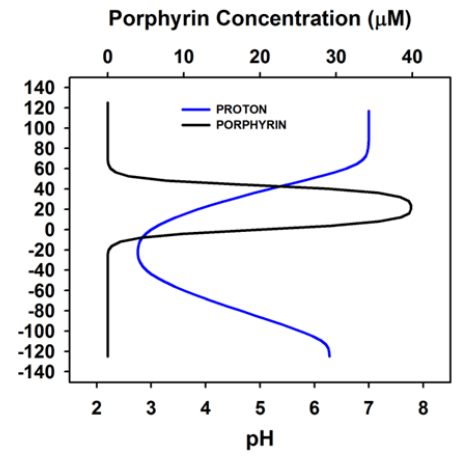

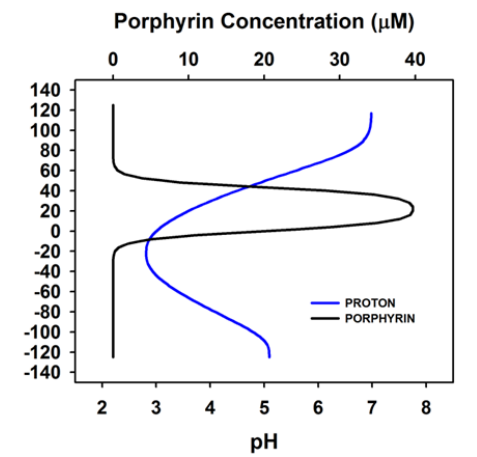

B
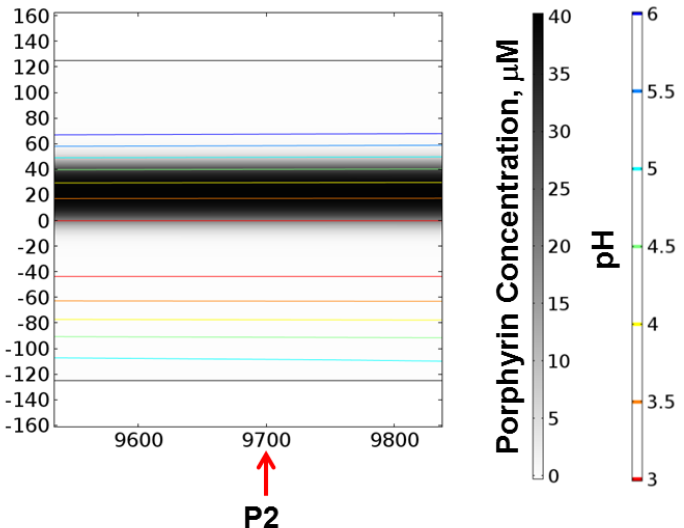

Figure S4. (A) 2D finite element simulations showing the diffusion of 1 (black color) and proton (colored lines) at P1 (left image), P2 (middle image) and P3 (right image). In (B), color legends for porphyrin concentration and $\mathrm{pH}$ are presented. In $(\mathrm{C})$, one-dimensional concentration profiles transversal to the direction of the flow, i.e. along the Y-coordinate axis, at P1, P2, and P3 are presented. The simulations show that laminar flow and the controlled diffusion mixing condition given by our microfluidic approach can lead to a precise porphyrin protonation inside the microfluidic channel. In this case, and in contrast to the simulation study shown in Figure S3 (C), the porphyrin can be partially protonated inside the microfluidic channel (at early stages of assembly) and under a controlled diffusion mixing process. 


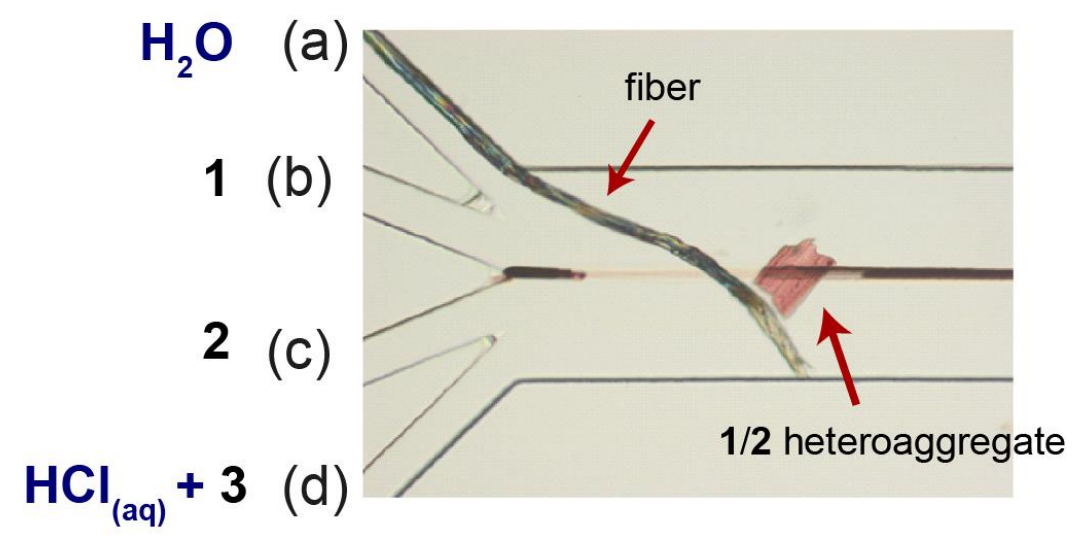

Figure S5. Optical microscope image of the four inlets and main microfluidic channel during the synthesis of M3. Accidentally a dust fiber entered into the main microfluidic channel through the inlet channel (a) breaking the sediment formed at the boundary between the 1-laden flow and the $\mathbf{2}$ stream. Note that the sediment starts to form at the upper and bottom layer of the microfluidic channel due to the non-slip boundary condition. The sediment produced consists of non-protonated porphyrin/surfactant heteroaggregates, previously described for $\mathbf{2 / 1}$ systems at neutral $\mathrm{pH}^{2}{ }^{2}$ The purple color of the porphyrin/surfactant heteroaggregates is typical for non-protonated $\mathbf{1}$. Therefore, this result is in agreement with the theoretical calculations presented in Figure $\mathrm{S} 3$ that confirm that when $\mathrm{HCl}_{(\mathrm{aq})}(2 \mathrm{mM})$ is injected in the lateral inlet channel (d), while $\mathbf{1}$ is injected in (b), the protonation of the porphyrin is avoided inside microfluidic channel. 

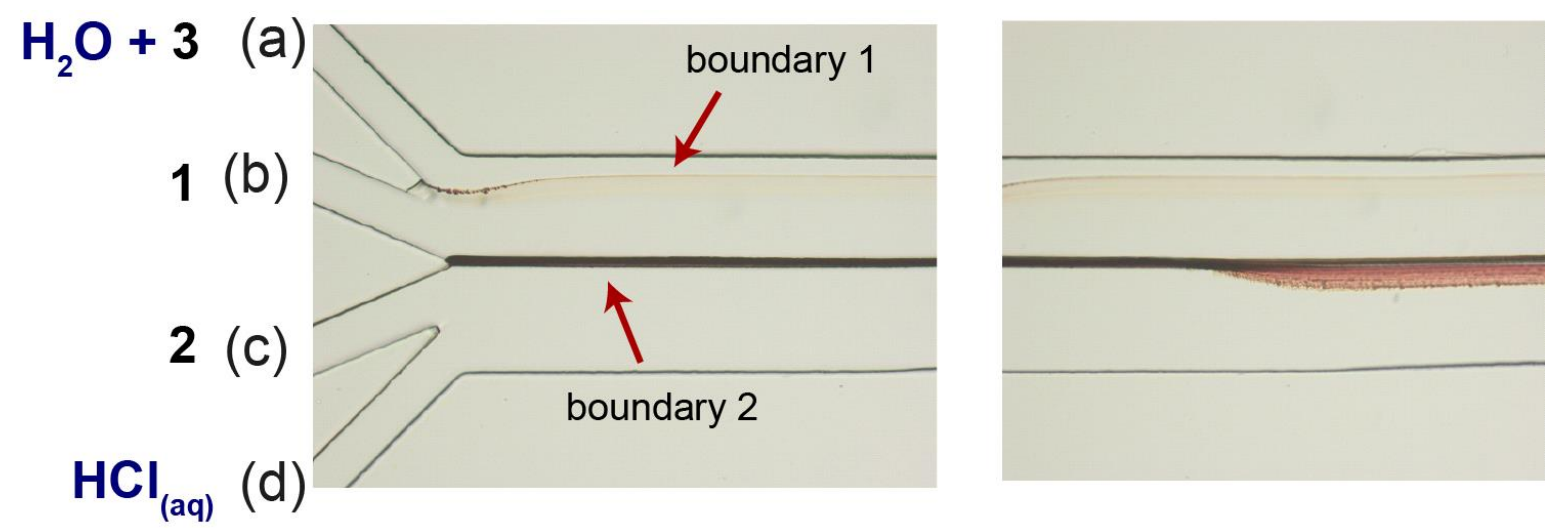

Figure S6. Micrograph of the four inlets and main microfluidic channel during the synthesis of M4. In this configuration, both the formation of $\mathbf{2 / 1}$ and $\mathbf{3 / 1}$ heteroaggregates occur, as evidenced by the sediments formed at the respective flow boundaries. 


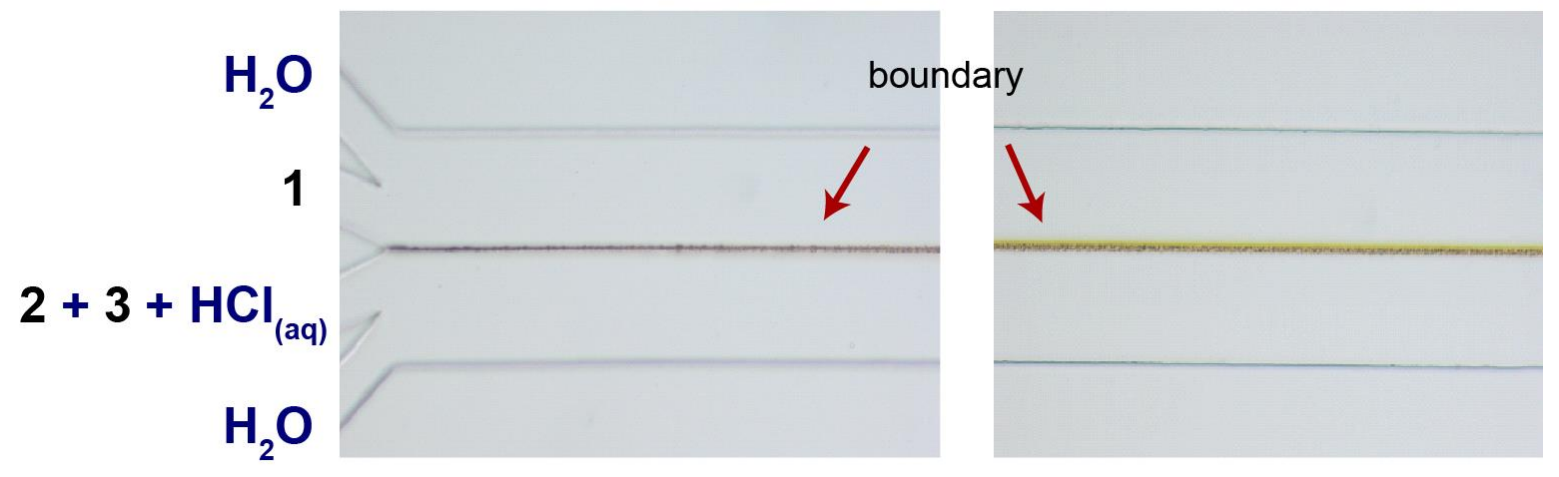

Figure S7. Micrograph of the four inlets and main microfluidic channel during a microfluidic mixing experiment in which 3 is injected together with 2 and $\mathrm{HCl}_{(\mathrm{aq})}(2 \mathrm{mM})$ in the central inlet channel (c) and adjacent to $\mathbf{1}$, injected in (b), i.e. M5 is synthesized. In this case, the formation of porphyrin/surfactant heteroaggregates involving protonated 1 occurs, as evidenced by the yellow-green residue shown in the optical microscope image acquired close to the microfluidic channel outlet (right micrograph). 
Section 5. Experiments in the presence of the chiral dopant 3; optimized microfluiding mixing (2/3 at ratio twenty to 0.75 )

A

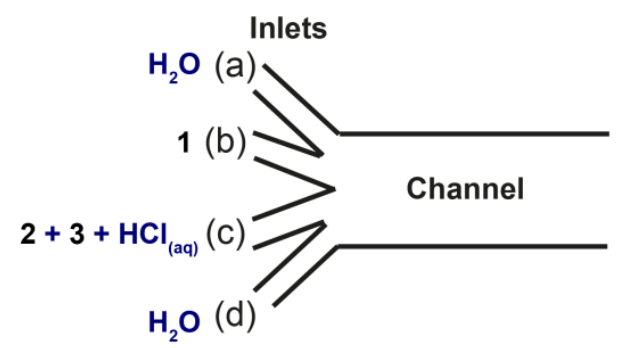

B

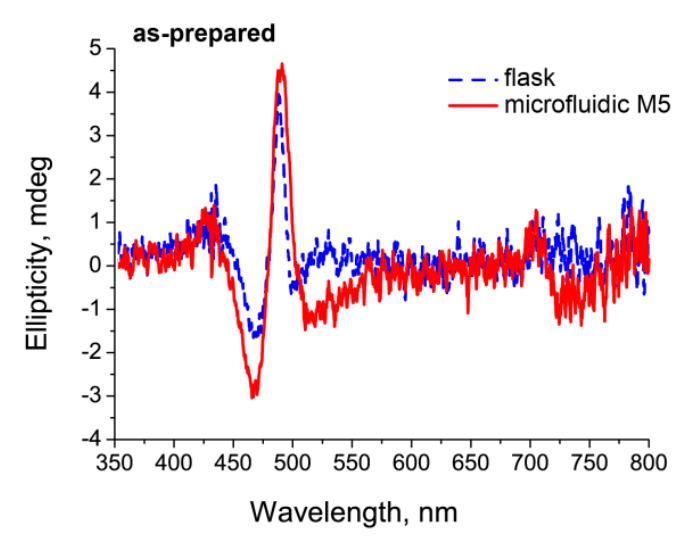

D

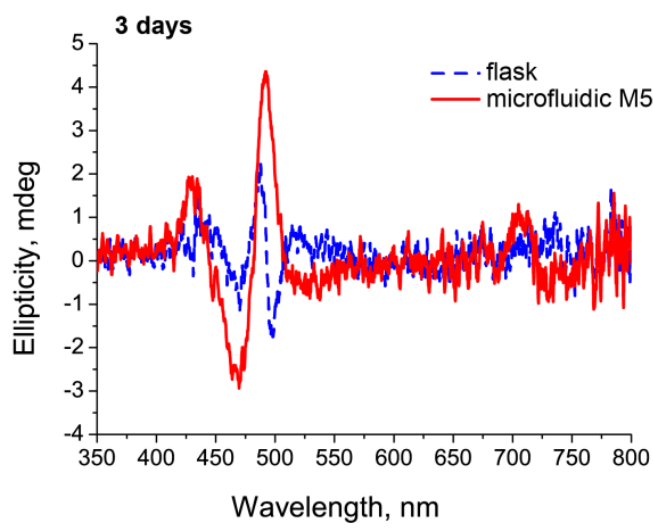

C

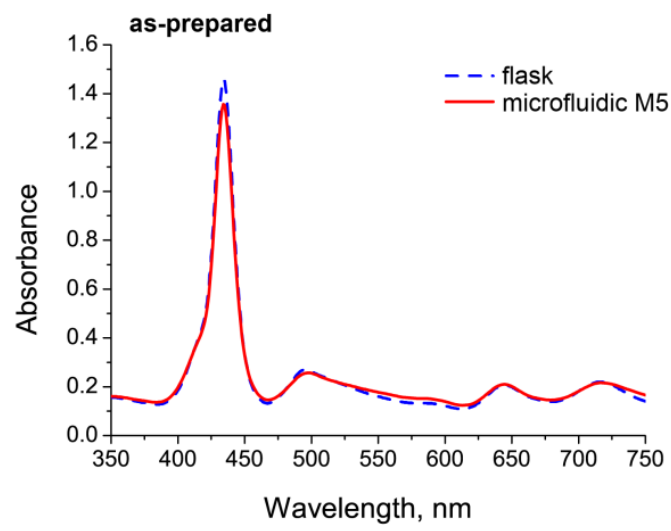

E

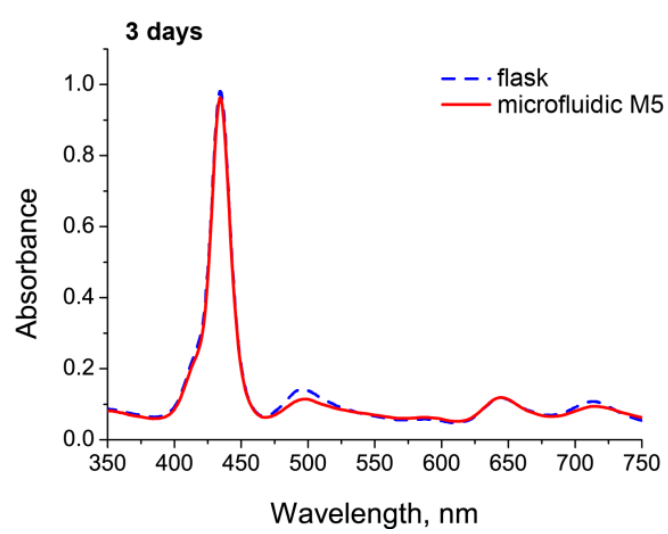

Figure S8. (A) Optimized configuration for the microfluidic mixing experiments, in which $\mathbf{3}$ is injected together with 2 and $\mathrm{HCl}_{(\mathrm{aq})}(2 \mathrm{mM})$ in the central inlet channel (c), adjacent to the 1-laden flow injected in (b), while two sheath water streams are injected in (a) and (d) inlet channels, i.e. M5 samples are synthetized. (B) and (C) are respectively the CD and UV-Vis spectra comparing an as-prepared microfluidic sample (prepared as described in (A)) with a flask sample prepared as described in Section 1. The same solutions used in the microfluidic mixing experiment described in (A) were used in the flask sample. (D) and (E) are the CD and UV-Vis spectra of the same samples after 2 days ageing (2/3 at ratio twenty to 0.75 , surfactants/1 ratio two to one, $[\mathbf{1}]=10 \mu \mathrm{M}$, optical path $=1 \mathrm{~cm}$ ). Only the CD spectrum of the flask sample evolved with time yielding a negative couplet analogous to the case showed in Figure 2 in the main text. 


\section{References}

(1) McDonald, J. C.; Whitesides, G. M. Acc. Chem. Res. 2002, 35, 491.

(2) Maiti, N. C.; Mazumdar, S.; Periasamy, N. J. Phys. Chem. B 1998, 102, 1528.

(3) Soudijn, Maarten L. Proton transport in aqueous ionic solutions. Diss. Master's thesis, University of Amsterdam, 2012.

(4) Da Costa, V. C. P.; Ribeiro, A. C. F.; Sobral, A.; Lobo, V. M. M.; Annunziata, O.; Santos, C.; Willis, S. A.; Price, W. S.; Esteso, M. A. J. Chem. Thermodyn. 2012, 47, 312.

(5) Gandini, S. C. M.; Yushmanov, V. E.; Borissevitch, I. E.; Tabak, M. Langmuir 1999, $15,6233$.

(6) Numata, M. Chem. - Asian J. 2015, 10, 2574. 